\title{
Erratum
}

\section{Erratum to: The Adolescent and Young Adult Self-Management and Independence Scale (AMIS-II): Expanding evidence for validity and reliability}

Monique M. Ridosh, Colleen Stiles-Shields, Alexa Stern, Adrien M. Winning, Lara Anderson, Kathleen J. Sawin and Grayson N. Holmbeck

[Journal of Pediatric Rehabilitation Medicine, 14(4), 2021, 583-596. DOI 10.3233/PRM-200679]

https://content.iospress.com/articles/journal-of-pediatric-rehabilitation-medicine/prm200679

On page 590, under the topic 3.3, there is a decimal missing. The section should read as follows:

\subsection{Internal consistency reliability analyses}

Overall, the AMIS-II demonstrated adequate internal consistency reliability. Internal consistency reliabilities for the AMIS-II total scale and subscales were high $(\alpha=0.90-0.95)$. In the Hispanic/Latino subsample, the internal consistency reliabilities were also high for the AMIS-II total scale $(\alpha=0.94)$ and the subscales $(\alpha=0.88-0.92)$. 